\title{
Targeting Wideband Circular Polarization: An Efficient $2 \times 2$ Sequentially-Phase-Fed Rotated Array Antenna
}

\author{
Saeed MOHAMMADI-ASL ${ }^{1}$, Javad NOURINIA ${ }^{1}$, Changiz GHOBADI ${ }^{1}$, Maryam MAJIDZADEH ${ }^{2}$ \\ ${ }^{1}$ Electrical Engineering Dept., Urmia University, Urmia, Iran \\ ${ }^{2}$ Dept. of Electrical and Computer Engineering, Urmia Girls Faculty, West Azarbaijan branch, Technical and Vocational \\ University (TVU), Urmia, Iran
}

aslelectco@gmail.com,J.Nourinia@urmia.ac.ir, Ch.Ghobadi@urmia.ac.ir,mmajidzadeh@tvu.ac.ir

Submitted May 16, 2017 / Accepted October 18, 2017

\begin{abstract}
This paper outlines an efficient design of a wideband sequential-phase (SP) fed circularly polarized corner-truncated patch array antenna (CPCTPAA) with sequentially rotated (SR) array elements. The SP feed network is composed of a circular ring with an inner section of $270^{\circ}$. Four shorting strips are attached to the circular ring to the end of which corner-truncated patches (CTPS) are attached. Through the proposed feed structure, stable phase difference is attained at the end of the shorting strips which in turn ends in CP generation. Each array element of $2 \times 2$ CPCTPAA is a CTP printed on the top side of the FR4 substrate. On the backside, rectangular slots are removed from the ground plane to enhance the bandwidth characteristics of the proposed design. Moreover, suitable rectangular slots are embedded at the crossing points of CTPs and feed lines which instigate extra improvements in СРСТРАA performance. These amendments, all together, are deemed for achieving an efficient and cost-effective CPCTPAA design with smaller occupied implementation space. Simulation and experimental studies are conducted on a $70 \times 70 \times 1.6 \mathrm{~mm}^{3}$ prototype to evaluate performance of the proposed array antenna. Results are discussed in depth.
\end{abstract}

\section{Keywords}

Circular polarization, corner-truncated patches, rectangular slots, sequential phase feed, wide impedance bandwidth, wide $\mathrm{CP}$ bandwidth

\section{Introduction}

Now, there is a commonsense regarding the marvelous merits of circularly polarized (CP) antennas which has stimulated a swift progress of this category over the linearly polarized ones. Some of these spectacular advantages are the ability to overcome multipath fading problem, performance independency to the path between transmitter and receiver, and the ability of withstanding against the unsuitable weather conditions. Specifically speaking, CP is generated when two orthogonal electric filed vectors are excited simultaneously. In the literature, different techniques have been proposed to excite these orthogonal vectors and stimulate CP feature [1-5]. Although contributing to significant improvements and applicable methods, there are still challenges in design of $\mathrm{CP}$ antennas regarding the $\mathrm{CP}$ purity, size reduction, and further performance enhancement.

On the plus side, array antennas have gained great popularity in recent years to enhance the overall performance of the proposed designs. Array antenna is defined as a combination of single antenna elements working together as a unit. In these antennas, well-arranged single antenna arrays bring about considerable enhancements regarding different features. It is worth mentioning that array type, element type, elements spacing and configuration, and feed network structure are some of the crucial issues impacting the overall triumph of established design. Some recent studies on array antenna structures are reported in [6-9].

Being inspired by the advantages of both $\mathrm{CP}$ and array antennas, CP array antennas (CPAAs) are perceived as one of the most applicable antenna categories. High gain, improved performance, and wide impedance and AR bandwidth are some of the CPAAs prominent features. Here, the feed network configuration plays an important role in design of efficient CPAAs. So far, many techniques have been adopted to excite CPAAs. Sequential phase (SP) feeding strategy is a promising approach in which stable phase difference is generated on radiating elements due to which, CP is achieved. A brief literature review reveals that well-designed SP feed network could significantly enhance performance of $2 \times 2$ CPAAs [10-13].

Targeting further improvements in overall performance of the established design, this paper aims at designing an efficient sketch of a CPAA with enhanced features. The established $2 \times 2$ array antenna is composed of a simple ground plane with four rectangular slots, four corner truncated patches (CTPs) as the radiating elements, and a novel SP feed network. The SP feed configuration consists of a circular ring, a sector of $270^{\circ}$, and four shorting strips. The CTPs are connected to the end of the shorting strips in 
sequentially rotated (SR) configuration. Moreover, rectangular slots are created at the crossing points of the SP feed and CTPs to further enhance the anticipated performance. As the SP feed strategy is adopted, stable phase difference is reflected at the end of the shorting strips. Consequently, the same phase difference is observed on CTPs. The proposed circularly polarized corner-truncated patch array antenna (CPCTPAA) design reveals a simple, compact, and cost-effective design with wide operating bandwidth. The substrate of the proposed design is based on FR4 material with thickness of $1.6 \mathrm{~mm}$ and loss tangent of 0.02 . A set of simulated and experimental analysis are conducted to assess performance of the proposed design in accordance with the expected features. Results well-support the main contributions of the present study revealed as follows.

- Proposing an efficient sketch of a $2 \times 2$ CPCTPAA with wide $10-\mathrm{dB}$ impedance and $3-\mathrm{dB}$ AR bandwidth through a smaller size against the similar designs.

- Utilizing simple CTPs in SR to be in line with technoeconomic objectives.

- Implementation of an efficient SP feed network with further performance enhancements.

This paper is organized as follows: Section 2 discusses the design and performance of the proposed $2 \times 2$ CPCTPAA. Here, 10-dB impedance and 3-dB AR bandwidth enhancements are discussed in detail. As well, the configuration and operating mechanism of the SP feed network is carefully explored. Section 3 addresses the measured results of the fabricated experimental prototype. Section 4 establishes a comparative study with the aim of shedding lights on the advantages of the proposed design. Ultimately, Section 5 concludes the paper.

\section{2. $2 \times 2$ CPCTPAA Configuration, Design, and Performance}

A well-selected feed network is shown to remarkably impact the overall performance of an antenna design. Accordingly, a dedicated effort is put on establishing efficient feed strategy for the proposed CPCTPAA. Afterwards, the design procedure of the $2 \times 2$ array is tailored in detail. Figure 1 depicts the proposed SP feed network configuration. As can be seen, the constituting elements are a circular ring, a sector of $270^{\circ}$, and four shorting strips. The outer and inner radii of the circular ring and sector of $270^{\circ}$ are $r_{1}$, $r_{2}, r_{3}$, and $r_{4}$ respectively. It is shown that the circular ring and sector of $270^{\circ}$ are connected to each other via a simple rectangular element with length and width of $\mathrm{L}_{1}$ and $\mathrm{W}_{1}$ respectively. As well, four shorting strips with the length of $\mathrm{L}_{2}$ and width of $\mathrm{W}_{2}$ are connected to the circular ring element. The feeding configuration is printed on $1.6 \mathrm{~mm}$ thick FR4 substrate with permittivity of 4.4 and loss tangent of 0.02 . As clarified, adoption of SP feed in arrays is a technique to enhance the impedance and AR bandwidth. Accordingly, a similar approach is adopted in the proposed

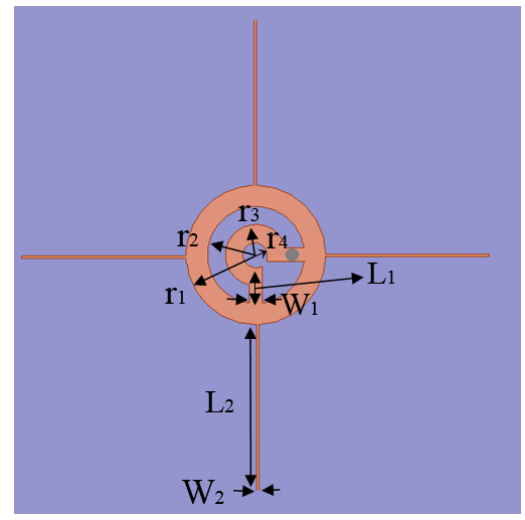

Fig. 1. Configuration and dimensions of the proposed SP feed network.

\begin{tabular}{|c|c|c|c|}
\hline $\mathrm{W}_{1}$ & $\mathrm{~L}_{1}$ & $\mathrm{~W}_{2}$ & $\mathrm{~L}_{2}$ \\
\hline 1.3 & 3.6 & 0.2 & 14.5 \\
\hline \hline $\mathrm{r}_{1}$ & $\mathrm{r}_{2}$ & $\mathrm{r}_{3}$ & $\mathrm{r}_{4}$ \\
\hline 6.8 & 4.8 & 3 & 1.3 \\
\hline
\end{tabular}

Tab. 1. Detailed values of the SP feed parameters (all values are in $\mathrm{mm}$ ).

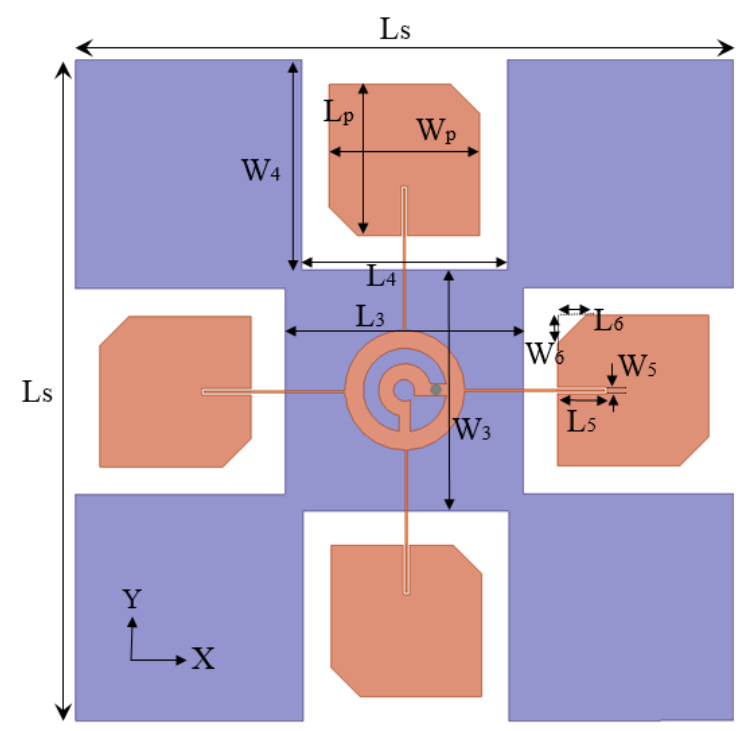

Fig. 2. $2 \times 2$ CPCTPAA structure and dimensions.

\begin{tabular}{|c|c|c|c|}
\hline $\mathrm{L}_{\mathrm{s}}$ & $\mathrm{W}_{\mathrm{p}, \mathrm{Lp}}$ & $\mathrm{W}_{3}, \mathrm{~L}_{3}$ & $\mathrm{~W}_{4}$ \\
\hline 70 & 16 & 23.5 & 23.2 \\
\hline \hline $\mathrm{L}_{4}$ & $\mathrm{~W}_{5}$ & $\mathrm{~L}_{5}$ & $\mathrm{~W}_{6,} \mathrm{~L}_{6}$ \\
\hline 22 & 3 & 1.3 & 2.8 \\
\hline
\end{tabular}

Tab. 2. Detailed values of the $2 \times 2$ CPCTPAA parameters (all values are in $\mathrm{mm}$ ).

design. Detailed values of the feed network parameters are reported in Tab. 1. All the values are in $\mathrm{mm}$.

Figure 2 illustrates the structure of the proposed $2 \times 2$ CPCTPAA. As can be seen, four elements are deployed in CPCTPAA structure. Each array element is composed of a CTP which is connected to the end of the shorting strip. The length and width of the CTPs are $L_{p}$ and $W_{p}$ respectively. Rectangular truncation with dimensions of $\mathrm{L}_{6}$ and $\mathrm{W}_{6}$ is created at opposite corners of each CTP. Besides, four rectangular slots with the length and width of $\mathrm{L}_{4}$ and 
$\mathrm{W}_{4}$ are removed from the ground plane to further enhance the CPCTPAA performance. What is more, rectangular slots are embedded on top side of the substrate at crossing points of the CTPs and shorting strips of the feed structure. The width and length of the conductive element inside the CTP are $\mathrm{W}_{5}$ and $\mathrm{L}_{5}$, respectively. As well, elements spacing is denoted by $\mathrm{W}_{3}$ and $\mathrm{L}_{3}$ which are both tuned at $23.5 \mathrm{~mm}$. Conducted studies in [10], [14] reveal that the optimal element spacing is obtained in $0.7 \lambda_{\mathrm{g}}$, where $\lambda_{\mathrm{g}}$ is the guided wavelength. In commonly used substrates with low dielectric constant between 3 and 5 , a general resonant type $\mathrm{CP}$ antenna has a size of about a half-wavelength and a margin of $0.1 \lambda_{\mathrm{g}}$ for the distance between SP feed and the antenna element. Moreover, the desired size of a SP feed should be evaluated at $0.3-0.4 \lambda_{\mathrm{g}}[10]$, [14]. The proposed CP array antenna is in line with the abovementioned metrics to avoid the possible side effects of a non-suitable elements spacing. Detailed values of the CPCTPAA parameters are reported in Tab. 2.

To provide an accurate investigation of the $2 \times 2$ CPCTPAA design and performance procedure, a step-bystep analysis is launched in Fig. 3. To investigate the effect of truncating the patch corners, in step 1 , simple square patches without any corner truncation are included and excited by the proposed SP feed network. In step 2, by truncating the corners, simple CTPs are deployed in array structure. No slots are adopted neither on the ground plane nor on the jointing points of the feed lines and CTPs. In step 3, rectangular slots are embedded on the ground plane. However, no slots are included on the conductive CTPs. In step 4 which represents the final structure, suitable slots are included on both of the ground plane and the CTPs.

Each of the aforementioned configurations is simulated using Ansoft High Frequency Structure Simulator (HFSS) and the corresponding $\mathrm{S}_{11}$ and AR curves are displayed in Figs. 4 and 5, respectively. It is seen that in step 1, when there are no truncation on patches, the frequency band of 5.4-6.3 GHz is covered by the array antenna. This is why no circular polarization is obtained. Then in step 2, by truncating the corners of the patches and without any slots, two resonances are excited at $5.7 \mathrm{GHz}$ and $6.4 \mathrm{GHz}$. In this step, the frequency band of $5.5-6.4 \mathrm{GHz}$ is covered by the CPCTPAA and CP is seen at 5.8-6.2 GHz. Hence, the effect of truncating the patches corners is directly observed in impedance bandwidth enhancement and more importantly in $\mathrm{CP}$ generation mechanism. By cutting rectangular slots on the ground plane in step 3,10-dB impedance bandwidth of $4.9-7.1 \mathrm{GHz}$ and 3-dB AR bandwidth of 5.2-5.4 and 6-6.4 GHz are observed. It is clearly seen that due to the coupling effects between the ground plane slots and CTPs, the excited resonances in step 2 shift toward lower and higher frequencies which extends the impedance bandwidth. Also, there is a gap between the two frequency bands in AR bandwidth. It is worth mentioning that although including ground plane slots extends the impedance bandwidth, generation of bi-directional radiation could be a possible side effect which deteriorates the radiation properties. Hence, by wise tuning of the slots dimensions and providing a trade-off between the bandwidth and radiation

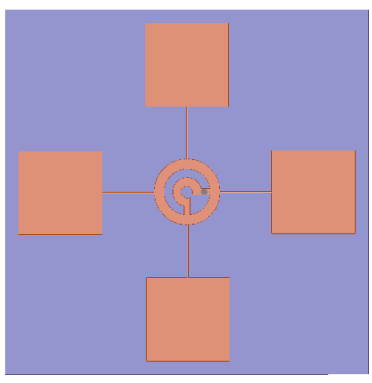

(a)
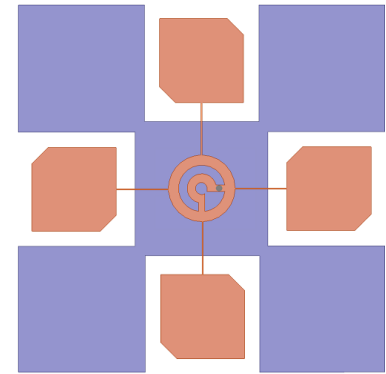

(c)

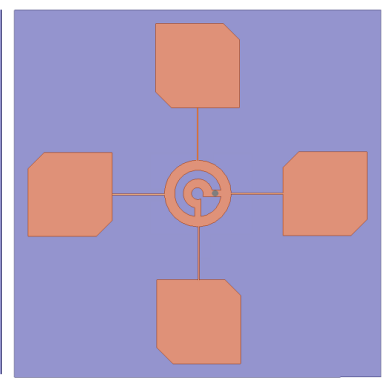

(b)

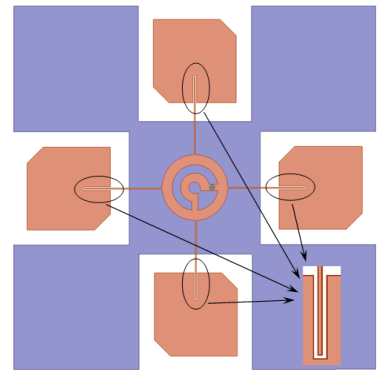

(d)
Fig. 3. Design steps of the proposed $2 \times 2$ CPCTPAA: (a) step 1, (b) step 2, (c) step 3, and (d) step 4.

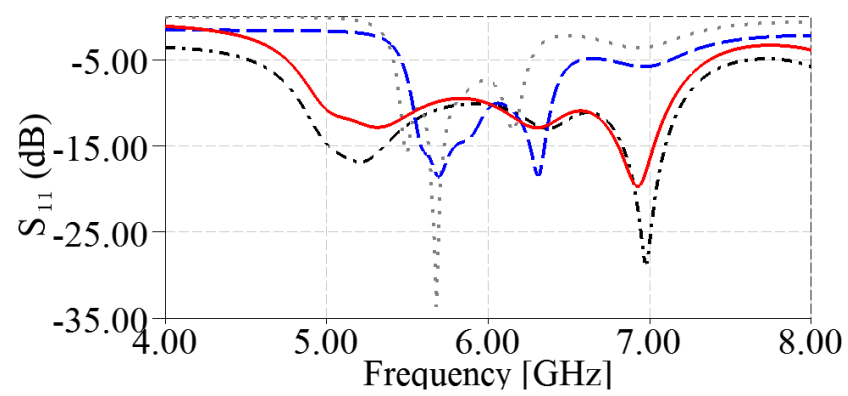

simple array

- - array with truncated patches, without slots on the ground plane and patches _ array without slots on the patches

-... proposed $2 \times 2$ array

Fig. 4. $S_{11}$ curves at four design steps of the proposed $2 \times 2$ CPCTPAA.

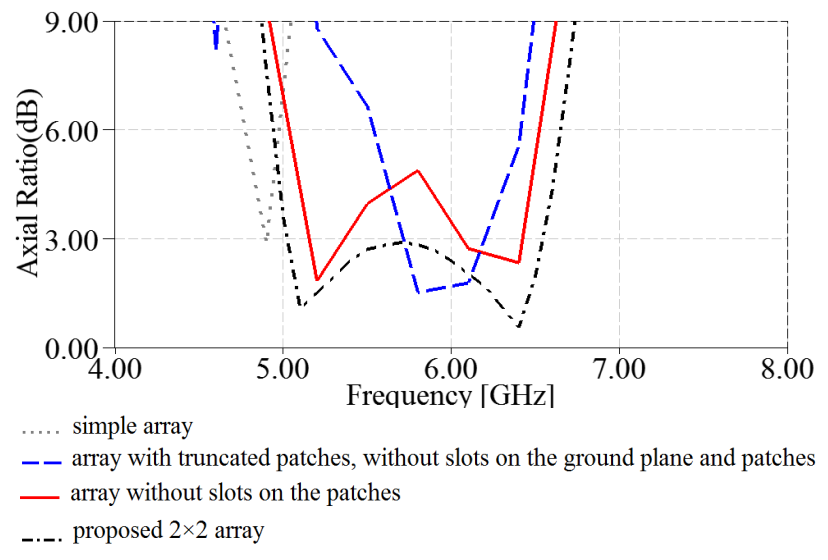

Fig. 5. AR curves at four design steps of the proposed $2 \times 2$ CPCTPAA.

pattern, the possibility of having such a condition is greatly lessened. In step 4 which depicts the final array configuration, by embedding the rectangular slots on CTPs, no 
changes are observed in 10-dB impedance bandwidth. This observation is due to the fact that addition of these slots does not introduce any new mode, but modifies the existing ones [15]. The operating impedance bandwidth is from 4.85 to $7.2 \mathrm{GHz}$. Moreover, CP feature is provided in $5-6.6 \mathrm{GHz}$. The obtained results in steps 3 and 4 clearly demonstrate that the inclusion of slots does not stimulate new modes. However, it modifies higher order orthogonal mode resonance frequency of the microstrip antennas.

According to the resonance frequency formulations in (1), the resonance frequency is a function of $L_{\mathrm{e}}$ and $\varepsilon_{\mathrm{re}}$ which in turn are dependent on slots locations and dimensions [15]

$$
f=\frac{c}{2 L_{\mathrm{e}} \sqrt{\varepsilon_{\mathrm{re}}}} .
$$

To further analyze the $2 \times 2$ CPCTPAA performance, surface current distribution is explored in Fig. 6. The results indicate that the current magnitudes are equal in $0^{\circ}$, $90^{\circ}, 180^{\circ}$, and $270^{\circ}$. However, the current phases at $0^{\circ}$ and $180^{\circ}$ oppose to those of $90^{\circ}$ and $270^{\circ}$. This observation is in line with right-handed $\mathrm{CP}$ (RHCP) in $+z$ direction and left-handed CP (LHCP) in-z direction. As clarified earlier, in SP feed network, stable phase difference is generated at the end of the four shorting strips to the end of which CTPs are connected. Hence, fixed current magnitude with stable phase difference yields in $\mathrm{CP}$ generation.

\section{Experimental Analysis: Results and Discussions}

To investigate performance of the proposed $2 \times 2$ CPCTPAA in real-world applications, an experimental prototype is fabricated and measured in antenna and microwave laboratory. The fabricated prototype, printed on $1.6 \mathrm{~mm}$ thick FR4 substrate, is shown in Fig. 7. Simulated and measured $\mathrm{S}_{11}$ curves of the proposed $2 \times 2$ CPCTPAA are depicted in Fig. 8. It is evidently seen that results are in

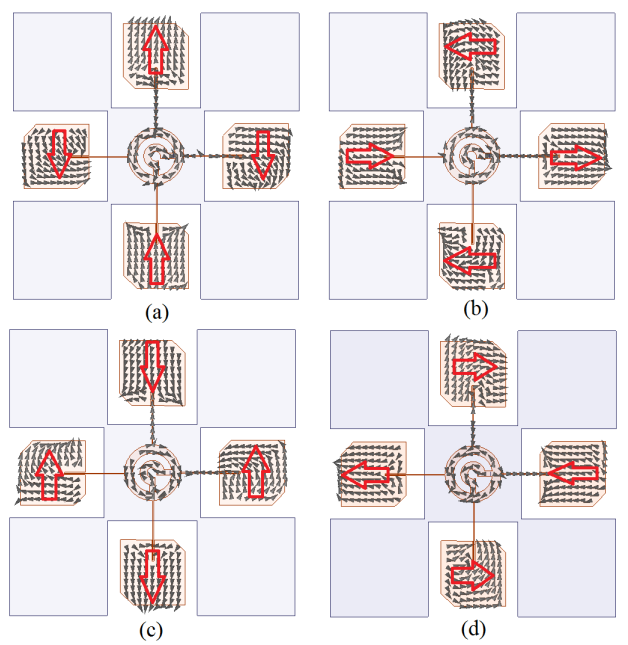

Fig. 6. Surface current distribution on the proposed $2 \times 2$ CPCTPAA: (a) $0^{\circ}$, (b) $90^{\circ}$, (c) $180^{\circ}$, and (d) $270^{\circ}$.

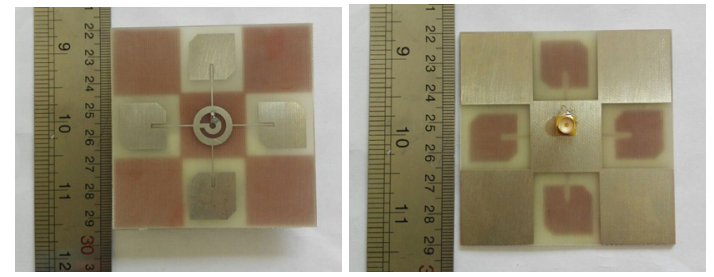

Fig. 7. Top and bottom view of the fabricated prototype of the proposed $2 \times 2$ CPCTPAA.

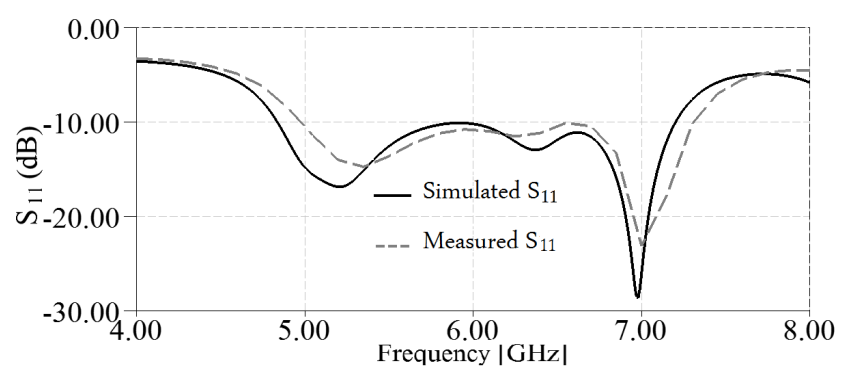

Fig. 8. Simulated and measured $S_{11}$ curves of the proposed $2 \times 2$ CPCTPAA.

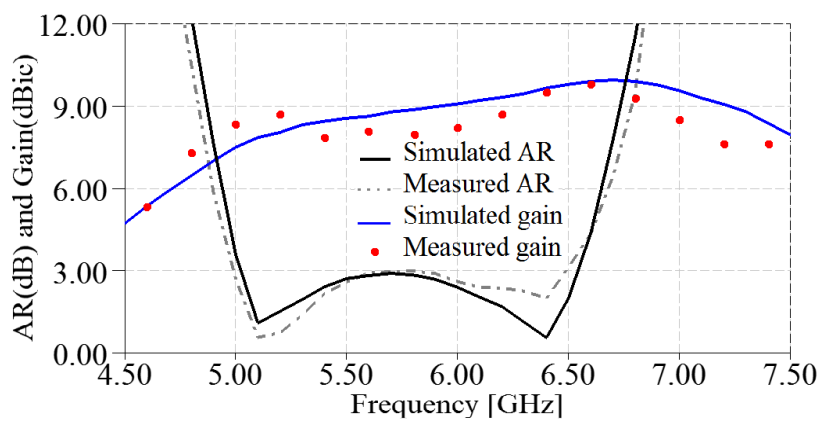

Fig. 9. Simulated and measured $A R$ and gain curves of the proposed $2 \times 2$ CPCTPAA.

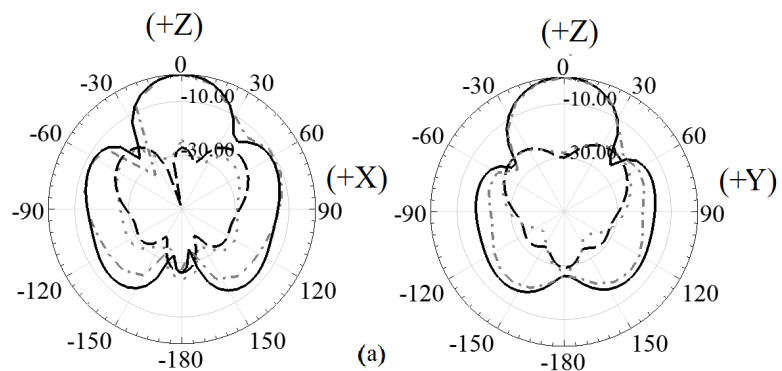

$(+Z)$

$(+Z)$

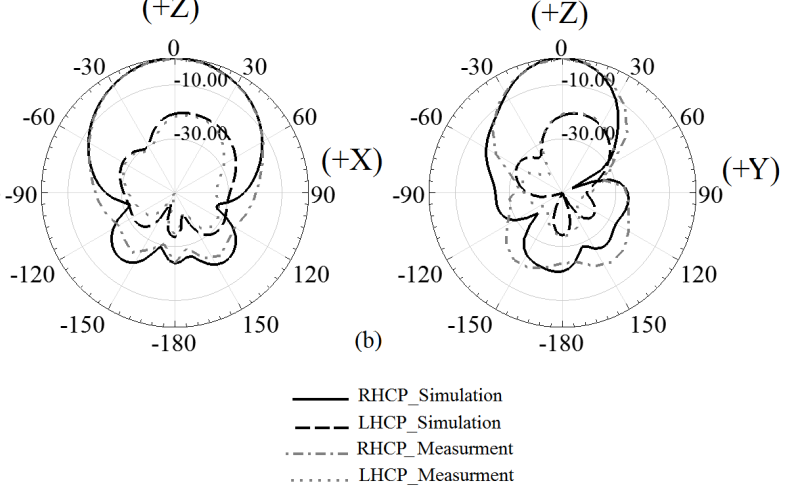

Fig. 10. Simulated and measured radiation patterns at: (a) $5.2 \mathrm{GHz}$ and (b) $6.4 \mathrm{GHz}$. 


\begin{tabular}{|c|c|c|c|c|}
\hline \multirow{2}{*}{$\begin{array}{c}\mathbf{2} \times \mathbf{2} \\
\text { arrays }\end{array}$} & \multicolumn{4}{|c|}{ Operational and structural characteristics } \\
\cline { 2 - 5 } & $\begin{array}{c}\text { Total size } \\
\left(\mathbf{m m}^{\mathbf{3}} \mathbf{)}\right.\end{array}$ & Feed network & $\begin{array}{c}\mathbf{1 0 - d B} \\
\text { impedance } \\
\text { BW }(\mathbf{G H z})\end{array}$ & $\begin{array}{c}\text { 3-dB AR } \\
\mathbf{B W}(\mathbf{G H z})\end{array}$ \\
\hline 10 & $140 \times 140 \times 0.6$ & $\begin{array}{c}\text { Single stage transi- } \\
\text { tion of impedance } \\
\text { transformers }\end{array}$ & $-2.42-2.6$ & $-2.45-2.57$ \\
\hline 11 & $100 \times 100 \times 3$ & $\begin{array}{c}\text { A loop with four } \\
\text { shorting strips }\end{array}$ & $4.82-5.12$ & $4.84-5.13$ \\
\hline 12 & $90 \times 90 \times 3$ & SP loop & $4.46-5.5$ & $4.52-5.4$ \\
\hline 13 & $75 \times 75 \times 1.5$ & $\begin{array}{c}\text { Loop and a sector of } \\
270^{\circ}\end{array}$ & $5.2-6.23$ & $5.25-5.95$ \\
\hline $\begin{array}{c}\text { This } \\
\text { work }\end{array}$ & $70 \times 70 \times 1.6$ & $\begin{array}{c}\text { Circular ring and } \\
\text { a sector of } 270^{\circ} \text { with } \\
\text { four shorting strips }\end{array}$ & $5-7.3$ & $5-6.6$ \\
\hline
\end{tabular}

Tab. 3. Operational and structural characteristics of $2 \times 2$ CPAAs.

good agreement with each other. The simulated $\mathrm{S}_{11}$ bandwidth is between $4.85-7.2 \mathrm{GHz}$ which validates the measured result extended from 5 to $7.3 \mathrm{GHz}$. Apart from $\mathrm{S}_{11}$, simulated and measured $\mathrm{AR}$ and gain values are also compared in Fig. 9. As can be seen, both simulated and measured AR bandwidths extend from 5 to $6.6 \mathrm{GHz}$. Gain values vary between 7 and $10 \mathrm{dBic}$ in the operating $\mathrm{CP}$ bandwidth. Moreover, the simulated and measured normalized radiation patterns at $5.2 \mathrm{GHz}$ and $6.4 \mathrm{GHz}$ are plotted in xy and yz plane in Fig. 10. Based on this figure, simulated results are well-matched with the measured results. Also, it can be seen that co-polarization gain values are higher than the cross polarizations.

\section{Comparative Studies with Similar Designs}

This section aims at comparing the proposed $2 \times 2$ CPCTPAA against some of the similar previous designs. To this end, total size, feed network structure, 10-dB impedance bandwidth, and 3-dB AR bandwidth of the array antennas in [10-13] and those of the present CPCTPAA are reported in Tab. 3. The provided data clearly indicate that the array structures in [10-13] occupy larger area with respect to the proposed CPCTPAA. This is why narrower 10-dB impedance and 3-dB AR bandwidths are attained. Different forms of SP feed are utilized in the aforementioned structures. For instance in [13] a similar SP feed network with square structure is proposed. Although the feeding configurations resemble to each other, but wise inclusion of well-tuned slots in the present structure yields performance enhancement with respect to the similar previously designed configurations.

Comparing the results of the array structures confirms superiority of the proposed CPCTPAA in terms of impedance and AR bandwidths with respect to the similar previous $2 \times 2$ arrays. Hence, the proposed CPCTPAA could be effectively deployed in relevant applications.

\section{Conclusion}

An efficient design of a $2 \times 2$ CPCTPAA was proposed and discussed in this manuscript. SP feed network was selected as the feeding structure to stimulate the array performance enhancement. The feed configuration was composed of a circular ring, a sector of $270^{\circ}$ inside the ring, and four shoring strips. Four CTPs were connected to the end of the shorting strips in SR form. Rectangular slots on the ground plane and also at the joining points of the shorting strips to the CTPs yielded further performance enhancement. By adoption of SP feed, stable phase differences were generated at the end of the shorting strips and also on CTPs which stimulated the CP. Simulated and measured results revealed that $10-\mathrm{dB}$ impedance bandwidth of $5-7.3 \mathrm{GHz}$ and $3-\mathrm{dB}$ AR bandwidth of $5-6.6 \mathrm{GHz}$ are covered by the CPCTPAA. Specifically speaking, wide impedance and AR bandwidth, small size, suitable gain, and simple structure are some of the notable features of the proposed CPCTPAA design which makes it a promising nominate for communication systems applications.

\section{References}

[1] CAO, Y. F., CHEUNG, S. W. YUK, T. I. Dual-cap mushroom-like metasurface used in $\mathrm{CP}$ reconfigurable monopole antenna for performance enhancement. IEEE Transactions on Antennas and Propagation, 2015, vol. 63, no. 12, p. 5949-5955, DOI: 10.1109/TAP.2015.2489682

[2] NOGHABAEI, S. M., H., RAHIM, S. K. A., SOH, P. J., et al. A dual-band circularly polarized patch antenna with a novel asymmetric slot for WiMAX application. Radioengineering, 2013, vol. 22 , no. 1, p. 291-295.

[3] ZUO, S. L., YANG, L., ZHANG, Z. Y. Dual-band CP antenna with a dual-ring cavity for enhanced beamwidth. IEEE Antennas and Wireless Propagation Letters, 2015, vol. 14, p. 867-870. DOI: 10.1109/LAWP.2014.2382580

[4] ZHANG, L. DONG, T. Low RCS and high-gain CP microstrip antenna using SA-MS. Electronics Letters, 2017, vol. 53, no. 6, p. 375-376. DOI: 10.1049/el.2016.4654

[5] MANOHAR, M., KSHETRIMAYUM, R. S., GOGOI, A. K. A compact dual band-notched circular ring printed monopole antenna for super wideband applications. Radioengineering, 2017, vol. 26, no. 1, p. 64-70. DOI: 10.13164/re.2017.0064

[6] LEE, S. R., LIM, E. H., LO, F. L. Broadband single-layer E-patch reflect array. Radioengineering, 2017, vol. 26, no. 1, p. 97-106. DOI: 10.13164/re.2017.0097

[7] LIAO, S., XUE, Q. Compact UHF three-element sequential rotation array antenna for satcom applications. IEEE Transactions on Antennas and Propagation, 2017, vol. 65, no. 5, p. 2328-2338. DOI: 10.1109/TAP.2017.2684190

[8] WEI, K., LI, J. Y., WANG, L., et al. Study of horizontally polarized omnidirectional microstrip antenna arrays. Radioengineering, 2017, vol. 26, no. 1, p. 107-113. DOI: 10.13164/re.2017.0107

[9] KUZU, S., ALCAM, M. Array antenna using defected ground structure shaped with fractal form generated by Apollonius circle. IEEE Antennas and Wireless Propagation Letters. 2017, vol. 16, p. 1020-1023. DOI: 10.1109/LAWP.2016.2616944 
[10] LIN, S. K., LIN, Y. C. A compact sequential-phase feed using uniform transmission lines for circularly polarized sequential rotation arrays. IEEE Transactions on Antennas and Propagation, 2011, vol. 59, no. 7, p. 2721-2724. DOI: 10.1109/TAP.2011.2152346

[11] LI, Y., ZHANG, Z., FENG, Z. A sequential-phase feed using a circularly polarized shorted loop structure. IEEE Transactions on Antennas and Propagation, 2013, vol. 61, no. 3, p. 1443-1447. DOI: $10.1109 /$ TAP.2012.2227103

[12] YANG, W., ZHOU, J., YU, Z., et al. Bandwidth- and gainenhanced circularly polarized antenna array using sequential phase feed. IEEE Antennas and Wireless Propagation Letters, 2014, vol. 13, p. 1215-1218. DOI: 10.1109/LAWP.2014.2332560

[13] DENG, C., LI, Y., ZHANG, Z., et al. A wideband sequential-phase fed circularly polarized patch array. IEEE Transactions on Antennas and Propagation, 2014, vol. 62, no. 7, p. 3890-3893. DOI: $10.1109 /$ TAP.2014.2321380

[14] WILEY, A. R., GUO, Y. J. Circularly polarized ellipse-loaded circular slot array for millimeter-wave WPAN applications. IEEE Transactions on Antennas and Propagation, 2009, vol. 57, no. 10, p. 2862-2870. DOI: 10.1109/TAP.2009.2029305

[15] DESHMUKH, A. A., RAY, K. P. Formulation of resonance frequencies for dual-band slotted rectangular microstrip antennas. IEEE Antennas and Propagation Magazine, 2012, vol. 54, no. 4, p. 78-97. DOI: 10.1109/MAP.2012.6309159

\section{About the Authors ...}

Saeed MOHAMMADI-ASL was born in Urmia, Iran 1984. He received his B.Sc. and M.Sc. degrees in Electrical (Telecommunication) Engineering from Urmia University, Urmia, Iran in 2008 and 2010, respectively. His primary research interests are in antenna design, numerical methods in electromagnetic and microwave components.
Javad NOURINIA received his B.Sc. in Electrical and Electronic Engineering from Shiraz University, his M.Sc. degree in Electrical and Telecommunication Engineering from Iran University of Science and Technology, and his Ph.D. degree in Electrical and Telecommunication from University of Science and Technology, Tehran Iran in 2000. From 2000 he was an Assistant Professor and now he is a Professor in the Dept of Electrical Engineering of Urmia University, Urmia, Iran. His primary research interests are in antenna design, numerical methods in electromagnetic, microwave circuits.

Changiz GHOBADI was born in June, 1960 in Iran. He received his B.Sc. in Electrical Engineering Electronics, M.Sc. degrees in Electrical Engineering Telecommunication from Isfahan University of Technology, Isfahan, Iran and Ph.D. degree in Electrical-Telecommunication from University of Bath, Bath, UK in 1998. From 1998 he was an Assistant Professor and now he is a Professor in the Dept. of Electrical Engineering of Urmia University, Urmia, Iran. His primary research interests are in antenna design, radar and adaptive filters.

Maryam MAJIDZADEH was born in 1987 in Urmia, Iran. She received her B.Sc. in Electrical Engineering from Urmia University in 2009. Then, she received her M.Sc. and Ph.D. degrees in Communication Engineering from the same university in 2012 and 2016, respectively. She is now an Assistant Professor in the Dept. of Electrical and Computer Engineering, Urmia Girls Faculty, West Azarbaijan branch, Technical and Vocational University (TVU), Urmia, Iran. Her research interests include antenna design, antenna miniaturization techniques, frequency selective surfaces, and electromagnetic compatibility. 